\title{
Hermite Reduction and Creative Telescoping for Hyperexponential Functions
}

\author{
Alin Bostan ${ }^{1}$, Shaoshi Chen², Frédéric Chyzak ${ }^{1}$, Ziming Li ${ }^{3}$, Guoce Xin ${ }^{4}$ \\ ${ }^{1}$ INRIA, Palaiseau, 91120, (France) \\ ${ }^{2}$ Department of Mathematics, NCSU, Raleigh, 27695-8025, (USA) \\ ${ }^{3} \mathrm{KLMM}$, AMSS, Chinese Academy of Sciences, Beijing 100190, (China) \\ ${ }^{4}$ Department of Mathematics, Capital Normal University, Beijing 100048, (China) \\ schen21@ncsu.edu, \{alin.bostan, frederic.chyzak\}@inria.fr \\ zmli@mmrc.iss.ac.cn, guoce.xin@gmail.com
}

\begin{abstract}
We present a reduction algorithm that simultaneously extends Hermite's reduction for rational functions and the Hermite-like reduction for hyperexponential functions. It yields a unique additive decomposition and allows to decide hyperexponential integrability. Based on this reduction algorithm, we design a new method to compute minimal telescopers for bivariate hyperexponential functions. One of its main features is that it can avoid the costly computation of certificates. Its implementation outperforms Maple's function DEtools[Zeilberger]. Moreover, we derive an order bound on minimal telescopers, which is more general and tighter than the known one.
\end{abstract}

\section{Categories and Subject Descriptors}

I.1.2 [Computing Methodologies]: Symbolic and Algebraic Manipulation-Algebraic Algorithms

\section{General Terms}

Algorithms, Theory

\section{Keywords}

Hermite Reduction, Hyperexponential function, Telescoper

\section{INTRODUCTION}

Given a univariate rational function $r$, Hermite reduction in $[12,13,6]$ finds rational functions $r_{1}$ and $r_{2}$ s.t.

\footnotetext{
${ }^{*}$ S.C. was supported by the National Science Foundation (NSF) grant CCF-1017217, A.B. and F.C. were supported in part by the MSR-INRIA Joint Centre, Z.L. by two NSFC grants (91118001 and 60821002/F02), and G.X. by NSFC grant (11171231).
}

Permission to make digital or hard copies of all or part of this work for personal or classroom use is granted without fee provided that copies are not made or distributed for profit or commercial advantage and that copies bear this notice and the full citation on the first page. To copy otherwise, to republish, to post on servers or to redistribute to lists, requires prior specific permission and/or a fee.

Copyright 20XX ACM X-XXXXX-XX-X/XX/XX ...\$10.00. (i) $r=r_{1}+r_{2}$, (ii) $r_{1}$ is rational integrable, (iii) $r_{2}$ is a proper fraction with a squarefree denominator. The additive decomposition is unique, and $r$ is rational integrable if and only if $r_{2}=0$.

A univariate function is hyperexponential if its logarithmic derivative is rational. Exponential, radical and rational functions are hyperexponential. Rational Hermite reduction has been extended to hyperexponential functions by Davenport in [9] and by Geddes, Le and $\mathrm{Li}$ in [10]. The former aims at solving Risch's equation; the latter is a differential analogue of the reduction algorithm for hypergeometric terms in [2]. For a given hyperexponential function $H$, the reduction algorithms in $[9,10]$ compute two hyperexponential functions $H_{1}$ and $H_{2}$ s.t. (i) $H=H_{1}+H_{2}$, (ii) $H_{1}$ is hyperexponential integrable, (iii) $H_{2}$ is minimal in some sense. However, $\mathrm{H}_{2}$ is not unique in general and it may be nonzero even when $H$ is hyperexponential integrable. In order to decide the integrability of $H$, one additionally needs to compute polynomial solutions of a first-order linear differential equation.

The method of creative telescoping for hyperexponential functions is developed by Almkvist and Zeilberger in [3]. It is nowadays an important automatic tool for computing definite integrals. Recently, it has also played an important role in the resolution of intriguing problems in enumerative combinatorics $[14,15]$. For a bivariate hyperexponential function $H(x, y)$, the problem of creative telescoping is to find a nonzero operator $L\left(x, D_{x}\right) \in \mathbb{F}(x)\left\langle D_{x}\right\rangle$, the ring of linear differential operators over the rational-function field $\mathbb{F}(x)$, s.t.

$$
L\left(x, D_{x}\right)(H)=D_{y}(G)
$$

for some hyperexponential function $G$, where $D_{x}=\partial / \partial x$ and $D_{y}=\partial / \partial y$. The operator $L$ above is called a telescoper for $H$, and $G$ is the corresponding certificate. An algorithm for solving (1) is given in [3], and is based on differential Gosper's algorithm. An algorithm for rational-function telescoping is given in [5], and is based on Hermite reduction. The latter separates the computation for telescopers from that for certificates, and has a lower complexity than the former for rational functions.

In the present paper, we develop a reduction algorithm which, given a univariate hyperexponential function $H$, constructs two hyperexponential functions $H_{1}$ and $H_{2}$ s.t. (i) $H=H_{1}+H_{2}$, (ii) $H_{1}$ is hyperexponential integrable, and 
(iii) $H_{2}$ is either zero or not hyperexponential integrable. We show that $H_{2}$ in the above additive decomposition is unique and can be obtained without computing polynomial solutions of any differential equation. Our algorithm is based on the Hermite-like reduction in [10], a differential variant of the polynomial reduction in [2] and on the idea for reducing simple radicals in [18, Proposition 7]. The main new ingredients are the uniqueness of $H_{2}$ and an easy way to compute $\mathrm{H}_{2}$, which are crucial for many applications. These enable us to extend the reduction-based rational telescoping algorithm in [5] to the hyperexponential case, and derive an order bound on the telescopers. The bound is more general and tighter than that given in [4].

The rest of the paper is organized as follows. We review the notion of hyperexponential functions and Hermite-like reduction in Sections 2 and 3, respectively. A new reduction algorithm is developed for hyperexponential functions in Section 4. After introducing kernel reduction in Section 5, we present a reduction-based telescoping algorithm for bivariate hyperexponential functions, and derive an upper bound on the order of minimal telescopers in Section 6 . We briefly describe an implementation of the new telescoping algorithm, and present some experimental results in Section 7 , which validate its practical relevance.

As a matter of notation, we let $\mathbb{E}$ be a field of characteristic zero and $\mathbb{E}(y)$ be the field of rational functions in $y$ over $\mathbb{E}$. For a polynomial $p \in \mathbb{E}[y]$, we denote by $\operatorname{deg}(p)$ and $\operatorname{lc}(p)$ the degree and leading coefficient of $p$, respectively. Let $D_{y}$ denote the usual derivation $d / d y$ on $\mathbb{E}(y)$. Then $\left(\mathbb{E}(y), D_{y}\right)$ is a differential field.

\section{HYPEREXPONENTIAL FUNCTIONS}

Hyperexponential functions share the common properties of rational functions, simple radicals, and exponential functions. Together with hypergeometric terms, they are frequently viewed as a special and important class of "closedform" solutions of linear differential and difference equations with polynomial coefficients.

Definition 1. Let $\Phi$ be a differential field extension of $\mathbb{E}(y)$. A nonzero element $H \in \Phi$ is said to be hyperexponential over $\mathbb{E}(y)$ if its logarithmic derivative $D_{y}(H) / H \in \mathbb{E}(y)$.

The product of hyperexponential functions is also hyperexponential. Two hyperexponential functions $H_{1}, H_{2}$ are said to be similar if there exists $r \in \mathbb{E}(y)$ s.t. $H_{1}=r H_{2}$. The sum of similar hyperexponential functions is still hyperexponential, provided that it is nonzero.

For brevity, we use the notation $\exp \left(\int f d y\right)$ to indicate a hyperexponential function whose logarithmic derivative is $f$. For a rational function $r \in \mathbb{E}$, we have

$$
r \exp \left(\int f d y\right)=\exp \left(\int\left(f+D_{y}(r) / r\right) d y\right) .
$$

A univariate hyperexponential function $H$ is said to be $h y$ perexponential integrable if it is the derivative of another hyperexponential function. For brevity, we say "integrable" instead of "hyperexponential integrable" in the sequel.

Assume that $H=r \exp \left(\int f d y\right)$ is integrable. Then $H$ is equal to $D_{y}(G)$ for some hyperexponential function $G$. A straightforward calculation shows that $G$ is similar to $D_{y}(G)$, and so is $H$. Set $G=s \exp \left(\int f\right)$ for some $s \in \mathbb{E}(y)$. Then $H=D_{y}(G)$ if and only if

$$
r=D_{y}(s)+f s .
$$

Deciding the integrability of $H$ amounts to finding a rational solution $s$ s.t. the above equation holds.

\section{HERMITE-LIKE REDUCTION}

Reduction algorithms have been developed for computing additive decompositions of rational functions [16, 12, 13], hypergeometric terms $[1,2]$, and hyperexponential functions [9, 10]. Those algorithms can be viewed as generalizations of Gosper's algorithm [11] and its differential analogue [3, §5].

For a hyperexponential function $H$, a reduction algorithm computes two hyperexponential functions $H_{1}, H_{2}$ s.t.

$$
H=D_{y}\left(H_{1}\right)+H_{2} .
$$

It turns out that $H, H_{1}$ and $H_{2}$ are similar. So we may write $H=r \exp \left(\int f d y\right)$ and $H_{i}=r_{i} \exp \left(\int f d y\right)$, where $r, r_{i}, f$ belong to $\mathbb{E}(y)$ and $i=1,2$. Then (3) translates into

$$
r=D_{y}\left(r_{1}\right)+f r_{1}+r_{2}
$$

A reduction algorithm for computing (3) amounts to choosing rational functions $r, f$ and $r_{1}$ so that $r_{2}$ satisfies properties similar to those obtained in Hermite reduction for rational functions. There are at least two approaches to this end. One is given in [9], and the other in [10]. We review the latter, because the notion of differential-reduced rational functions plays a key role in Lemma 6 in Section 4.

Recall $[10, \S 2]$ that a rational function $r=a / b \in \mathbb{E}(y)$ is said to be differential-reduced w.r.t. $y$ if

$$
\operatorname{gcd}\left(b, a-i D_{y}(b)\right)=1 \quad \text { for all } i \in \mathbb{Z} .
$$

By Lemma 2 in [10], $r$ is differential-reduced if and only if none of its residues is an integer. The differential rational canonical form of a rational function $f$ in $\mathbb{E}(y)$ is a pair $(K, S)$ in $\mathbb{E}(y) \times \mathbb{E}(y)$ s.t. (i) $K$ is differential-reduced; (ii) the denominator of $S$ is coprime with that of $K$; and (iii) $f$ is equal to $K+D_{y}(S) / S$. Every rational function has a unique canonical form in the sense that $K$ is unique and $S$ is unique up to a multiplicative constant in $\mathbb{E}[10, \S 3]$. We call $K$ and $S$ the kernel and shell of $f$, respectively. They can be constructed by the method described in [10, §3].

Let $H$ be a univariate hyperexponential function in the form $\exp \left(\int f d y\right)$ over $\mathbb{E}$. Assume that $K$ and $S$ are the kernel and shell of $f$, respectively. Then $H=S \exp \left(\int K d y\right)$. Note that $K=0$ if and only if $H$ is a rational function, which is equal to $c S$ for some $c \in \mathbb{E}$.

Example 2. Let $H=\sqrt{y^{2}+1} /(y-1)^{2}$. The logarithmic derivative of $H$ is

$$
\frac{D_{y} H}{H}=\frac{D_{y}\left(1 /(y-1)^{2}\right)}{1 /(y-1)^{2}}+\frac{y}{y^{2}+1},
$$

where $y /\left(y^{2}+1\right)$ is differential-reduced. The kernel and shell of $D_{y}(H) / H$ are $y /\left(y^{2}+1\right)$ and $1 /(y-1)^{2}$, respectively. So $H=\exp \left(\int y /\left(y^{2}+1\right) d y\right) /(y-1)^{2}$.

For brevity, we make a notational convention.

Convention 3. Let $H$ denote a hyperexponential function whose logarithmic derivative has kernel $K$ and shell $S$. Assume that $K$ is nonzero, that is, $H$ is not a rational function. Set $T=\exp \left(\int K d y\right)$. Moreover, write $K=k_{1} / k_{2}$, where $k_{1}, k_{2}$ are polynomials in $\mathbb{E}[y]$ with $\operatorname{gcd}\left(k_{1}, k_{2}\right)=1$. 
The algorithm ReduceCert in [10] computes a rational function $S_{1}$ s.t.

$$
S=D_{y}\left(S_{1}\right)+S_{1} K+\frac{a}{b k_{2}}
$$

where $a, b \in \mathbb{E}[y]$ satisfy the following conditions: $b$ is the squarefree part of the denominator of $S$, and $\operatorname{gcd}\left(b, k_{2}\right)=1$. Note that $a$ is not necessarily coprime with $b k_{2}$. As the algorithm ReduceCert only reduces the shell $S$, it is referred to as the shell reduction. It follows from (4) that

$$
H=D_{y}\left(S_{1} T\right)+\frac{a}{b k_{2}} T .
$$

By Theorem 4 in [10], $a / b$ belongs to $\mathbb{E}[y]$ if $H$ is integrable.

Example 4. Let $H$ be the same hyperexponential function as in Example 2. Then $D_{y}(H) / H$ has kernel $K=y /\left(y^{2}+1\right)$ and shell $S=1 /(y-1)^{2}$. The shell reduction yields

$$
S=D_{y}\left(S_{1}\right)+S_{1} K+\frac{y}{(y-1) k_{2}},
$$

where $S_{1}=1 /(1-y)$ and $k_{2}=y^{2}+1$. Then $H$ can be decomposed into $H=D_{y}\left(S_{1} T\right)+y T /\left((y-1) k_{2}\right)$, where $T=\sqrt{y^{2}+1}$. By Theorem 4 in [10], $H$ is not integrable.

On the other hand, it is possible that $a$ in (5) is nonzero but $H$ is integrable.

Example 5. Let $H=y \exp (y)$ whose logarithmic derivative has kernel 1 and shell $y$, that is, $H=y \exp \left(\int 1 d y\right)$. But $H$ is integrable as it is equal to $D_{y}(y \exp (y)-\exp (y))$.

The shell reduction cannot be directly used to decide hyperexponential integrability. To amend this, the solution proposed in [10, Algorithm ReduceHyperexp] was to find the polynomial solutions of an auxiliary first-order linear differential equation. In the following section, we show how this can be avoided and improved.

\section{HERMITE REDUCTION FOR HYPEREXPONENTIAL FUNCTIONS}

After the shell reduction described in (5), the denominators of shells have been reduced to squarefree polynomials. In the rational case, i.e., when the kernel $K$ is zero, the polynomial $a$ in (4) can be chosen s.t. $\operatorname{deg}(a)<\operatorname{deg}(b)$, because all polynomials are rational integrable. But a hyperexponential function with a polynomial shell is not necessarily integrable. For example, $H=\exp \left(y^{2}\right)$.

We present a differential variant of $[2$, Theorem 7$]$ to bound the degree of $a$ in (4). The variant leads not only to a canonical additive decomposition of hyperexponential functions, but also a direct way to decide their integrability.

\subsection{Polynomial reduction}

With Convention 3, we define

$$
\mathcal{M}_{K}=\left\{k_{2} D_{y}(p)+k_{1} p \mid p \in \mathbb{E}[y]\right\} .
$$

It is an $\mathbb{E}$-linear subspace in $\mathbb{E}[y]$. We call $\mathcal{M}_{K}$ the subspace for polynomial reduction w.r.t. $K$. Moreover, define an $\mathbb{E}$ linear map $\phi_{K}$ from $\mathbb{E}[y]$ to $\mathcal{M}_{K}$ that, for every $p \in \mathbb{E}[y]$, maps $p$ to $k_{2} D_{y}(p)+k_{1} p$. We call $\phi_{K}$ the map for polynomial reduction w.r.t. $K$.

Concerning the subspace $\mathcal{M}_{K}$ and the map $\phi_{K}$, we have
Lemma 6. (i) If $k_{2} D_{y}(g)+k_{1} g \in \mathbb{E}[y]$ for some $g \in \mathbb{E}(y)$, then $g \in \mathbb{E}[y]$. (ii) The map $\phi_{K}$ is bijective.

Proof. Assume that $g$ has a pole. Without loss of generality, we assume that the pole is $y=0$ and has order $m$, because the following argument is also applicable over the algebraic closure of $\mathbb{E}$. Expanding $g$ around the origin yields

$$
g=\frac{r}{y^{m}}+\text { terms of higher orders in } y,
$$

where $r \in \mathbb{E} \backslash\{0\}$. It follows from $k_{2} D_{y}(g)+k_{1} g \in \mathbb{E}[y]$ that $y=0$ is a pole of

$$
\left(-\frac{m r}{y^{m+1}}+\text { higher terms }\right)+K\left(\frac{r}{y^{m}}+\text { higher terms }\right)
$$

with order no more than that of $K$. This implies that $y=0$ is a simple pole of $K$ with residue $m$, which is incompatible with $K$ being differential-reduced. The first assertion holds.

The map $\phi_{K}$ is surjective by its definition. If $\phi_{K}(p)=0$ for some nonzero polynomial $p \in \mathbb{E}[y]$, then $K$ equals $-D_{y}(p) / p$, which is nonzero since $K \neq 0$. So $K$ is not differentialreduced, a contradiction. The second assertion holds.

An $\mathbb{E}$-basis of $\mathcal{M}_{K}$ is called an echelon basis if distinct elements in the basis have distinct degrees. Echelon bases always exist and their degrees form a unique subset of $\mathbb{N}$. Let $\mathcal{B}$ be an echelon basis of $\mathcal{M}_{K}$. Define

$$
\mathcal{N}_{K}=\operatorname{span}_{\mathbb{E}}\left\{x^{\ell} \mid \ell \in \mathbb{N} \text { and } \ell \neq \operatorname{deg}(f) \text { for all } f \in \mathcal{B}\right\} .
$$

Then $\mathbb{E}[y]=\mathcal{M}_{K} \oplus \mathcal{N}_{K}$. We call $\mathcal{N}_{k}$ the standard complement of $\mathcal{M}_{K}$. Using an echelon basis of $\mathcal{M}_{K}$, one can reduce a polynomial $p$ to a unique polynomial $\tilde{p} \in \mathcal{N}_{K}$ s.t. $p-\tilde{p} \in \mathcal{M}_{K}$.

In order to find an echelon basis of $\mathcal{M}_{K}$, we set $d_{1}=\operatorname{deg} k_{1}$, $d_{2}=\operatorname{deg} k_{2}, \tau_{K}=-\operatorname{lc}\left(k_{1}\right) / \operatorname{lc}\left(k_{2}\right)$, and $\mathcal{B}=\left\{\phi_{K}\left(y^{n}\right) \mid n \in \mathbb{N}\right\}$. By Lemma 6 (ii), $\mathcal{B}$ is an $\mathbb{E}$-basis of $\mathcal{M}_{K}$. Let $p$ be a nonzero polynomial in $\mathbb{E}[y]$. We make the following case distinction.

Case 1. $d_{1} \geq d_{2}$. Then

$$
\phi_{K}(p)=\operatorname{lc}\left(k_{1}\right) \operatorname{lc}(p) y^{d_{1}+\operatorname{deg} p}+\text { lower terms. }
$$

So $\mathcal{B}$ is an echelon basis, in which $\operatorname{deg} \phi_{K}\left(y^{n}\right)=d_{1}+n$ for all $n \in \mathbb{N}$. Accordingly, $\mathcal{N}_{K}$ is spanned by $1, y, \ldots, y^{d_{1}-1}$.

Case 2. $d_{1}=d_{2}-1$ and $\tau_{K}$ is not a positive integer. Then $\phi_{K}(p)=\left(\operatorname{deg}(p) \operatorname{lc}\left(k_{2}\right)+\operatorname{lc}\left(k_{1}\right)\right) \operatorname{lc}(p) y^{d_{1}+\operatorname{deg} p}+$ lower terms.

Since $\tau_{K}$ is not a positive integer, $\operatorname{deg} \phi_{K}\left(y^{n}\right)=d_{1}+n$. Thus, $\mathcal{M}_{K}$ and $\mathcal{N}_{K}$ have the same bases as in Case 1.

Case 3. $d_{1}<d_{2}-1$. If $\operatorname{deg}(p)>0$, then

$$
\phi_{K}(p)=\operatorname{deg}(p) \operatorname{lc}\left(k_{2}\right) \operatorname{lc}(p) y^{d_{2}+\operatorname{deg}(p)-1}+\text { lower terms. }
$$

Otherwise, $\operatorname{deg} p=0$ and $\phi_{K}(p)=k_{1} p$. Therefore, $\mathcal{B}$ is again an echelon basis, in which

$\operatorname{deg} \phi_{K}(1)=d_{1}$ and $\operatorname{deg} \phi_{K}\left(y^{n}\right)=d_{2}+n-1$ for all $n \geq 1$. Accordingly, $\mathcal{N}_{K}$ has a basis $1, \ldots, y^{d_{1}-1}, y^{d_{1}+1}, \ldots, y^{d_{2}-1}$. Case 4. $d_{1}=d_{2}-1$ and $\tau_{K}$ is a positive integer. It follows from (6) that $\operatorname{deg} \phi\left(y^{n}\right)=d_{1}+n$ if $n \neq \tau_{K}$. Furthermore, for every polynomial $p$ of degree $\tau_{K}, \operatorname{deg}\left(\phi_{K}(p)\right)$ is of degree less than $d_{1}+\tau_{K}$. So any echelon basis of $\mathcal{M}_{K}$ does not contain a polynomial of degree $d_{1}+\tau_{K}$. Set

$$
\mathcal{B}^{\prime}=\left\{\phi\left(y^{n}\right) \mid n \in \mathbb{N}, n \neq \tau_{K}\right\}
$$


Reducing $\phi\left(y^{\tau_{K}}\right)$ by the polynomials in $\mathcal{B}^{\prime}$, we obtain a polynomial $r$ of degree less than $d_{1}$. Note that $r$ is nonzero, because $\mathcal{B}$ is an $\mathbb{E}$-linearly independent set. Hence, $\mathcal{B}^{\prime} \cup\{r\}$ is an echelon basis of $\mathcal{M}_{K}$. Consequently, $\mathcal{N}_{K}$ has an $\mathbb{E}$ basis $\left\{1, y, \ldots, y^{\operatorname{deg}(r)-1}, y^{\operatorname{deg}(r)+1}, \ldots, y^{d_{1}-1}, y^{d_{1}+\tau_{K}}\right\}$.

Example 7. Let $K=-6 y^{3} /\left(y^{4}+1\right)$, which is differentialreduced. Then $\tau_{K}=6$. According to Case $4, \mathcal{M}_{K}$ has an echelon basis $\{y\} \cup\left\{(n-6) y^{n+3}+n y^{n-1} \mid n \in \mathbb{N}, n \neq 6\right\}$. Moreover, $\mathcal{N}_{K}$ has a basis $\left\{1, y^{2}, y^{9}\right\}$.

One can reduce the degree and number of terms of a polynomial using a subspace of polynomial reduction.

Lemma 8. With Convention 3, we further let $d_{1}=\operatorname{deg} k_{1}$, $d_{2}=\operatorname{deg} k_{2}$, and $\tau_{K}=-\operatorname{lc}\left(k_{1}\right) / \operatorname{lc}\left(k_{2}\right)$. Let $\mathcal{M}_{K}$ be the subspace for polynomial reduction, and $\mathcal{N}_{K}$ its standard complement w.r.t. K. Finally, let $p$ be a polynomial in $\mathbb{E}[y]$.

(i) If $d_{1} \geq d_{2}$ or $d_{1}=d_{2}-1$ and $\tau_{K} \notin \mathbb{Z}^{+}$, then there exists $q \in \mathcal{N}_{K}$ s.t. $p \equiv q \bmod \mathcal{M}_{K}$ and $\operatorname{deg} q<d_{1}$.

(ii) If $d_{1}<d_{2}-1$, then there exists $q \in \mathcal{N}_{K}$ s.t. $p \equiv q$ $\bmod \mathcal{M}_{K}, \operatorname{deg} q<d_{2}$ and the coefficient of $y^{d_{1}}$ in $q$ is equal to zero.

(iii) If $d_{1}=d_{2}-1$ and $\tau_{K} \in \mathbb{Z}^{+}$, then there exists $r \in \mathbb{E}[y]$ of degree less than $d_{1}$ s.t.

$$
p \equiv s y^{d_{1}+\tau_{K}}+r \quad \bmod \mathcal{M}_{K} \quad \text { for some } s \in \mathbb{E} .
$$

Moreover, sy $y^{d_{1}+\tau_{K}}+r$ belongs to $\mathcal{N}_{K}$, and $r$ has at most $d_{1}-1$ terms.

Proof. The lemma is immediate from the $\mathbb{E}$-bases of $\mathcal{N}_{K}$ constructed in the above case distinction.

The next corollary enables us to derive an order bound on telescopers for hyperexponential functions.

Corollary 9. With the notation introduced in Lemma 8, there exists $\mathcal{P} \subset\left\{y^{n} \mid n \in \mathbb{N}\right\}$ with $|\mathcal{P}| \leq \max \left(d_{1}, d_{2}-1\right)$ s.t. every polynomial in $\mathbb{E}[y]$ can be reduced modulo $\mathcal{M}_{K}$ to an $\mathbb{E}$-linear combination of the elements in $\mathcal{P}$.

Proof. By the above case distinction, the dimension of $\mathcal{N}_{K}$ over $\mathbb{E}$ is at most $\max \left(d_{1}, d_{2}-1\right)$. The corollary follows.

\subsection{Hyperexponential integrability}

With Convention 3, we further assume that the polynomials $a$ and $b$ are obtained by the shell reduction in (5). So the decomposition (5) holds for the present notation. Moreover, let $\mathcal{M}_{K}$ be the subspace of polynomial reduction w.r.t. $K$, and $\mathcal{N}_{K}$ its standard complement.

We are going to determine necessary and sufficient conditions on hyperexponential integrability. Since $\operatorname{gcd}\left(b, k_{2}\right)=1$,

$$
\frac{a}{b k_{2}}=p+\frac{q}{b}+\frac{r}{k_{2}}
$$

where $p, q, r \in \mathbb{E}[y], \operatorname{deg}(q)<\operatorname{deg}(b)$, and $\operatorname{deg}(r)<\operatorname{deg}\left(k_{2}\right)$. Using an echelon basis of $\mathcal{M}_{K}$, we compute $u$ in $\mathcal{M}_{K}$ and $v$ in $\mathcal{N}_{K}$ s.t. $k_{2} p+r=u+v$. By the definition of $\mathcal{M}_{K}$, there exists $w$ in $\mathbb{E}[y]$ s.t. $u=k_{2} D_{y}(w)+k_{1} w$. By (7), we get

$$
\frac{a}{b k_{2}}=\frac{q}{b}+\frac{k_{2} D_{y}(w)+k_{1} w+v}{k_{2}}=D_{y}(w)+K w+\frac{q}{b}+\frac{v}{k_{2}} .
$$

It follows that

$$
\frac{a}{b k_{2}} T=D_{y}(w T)+\left(\frac{q}{b}+\frac{v}{k_{2}}\right) T .
$$

The process for obtaining (8) is referred to as the polynomial reduction for $\left(a /\left(b k_{2}\right)\right) T$ w.r.t. $K$, as it makes essential use of the subspaces $\mathcal{M}_{K}$ and $\mathcal{N}_{K}$. By (8) and (5),

$$
H=D_{y}\left(\left(S_{1}+w\right) T\right)+\left(\frac{q}{b}+\frac{v}{k_{2}}\right) T,
$$

which motivates us to introduce the notion of residual forms.

Definition 10. With Convention 3, we further let $f$ be a rational function in $\mathbb{E}(y)$. Another rational function $r \in \mathbb{E}(y)$ is said to be a residual form of $f$ w.r.t. $K$ if there exist $g$ in $\mathbb{E}(y)$ and $q, b, p$ in $\mathbb{E}[y]$ s.t.

$$
f=D_{y}(g)+K g+r \quad \text { and } \quad r=\frac{q}{b}+\frac{v}{k_{2}},
$$

where $b$ is squarefree, $\operatorname{gcd}\left(b, k_{2}\right)=1, \operatorname{deg} q<\operatorname{deg} b$, and $v$ is in the standard complement $\mathcal{N}_{K}$ of the subspace of polynomial reduction w.r.t. $K$. For brevity, we say that $r$ is a residual form w.r.t $K$ if $f$ is clear from context.

Residual forms are closely related to the integrability of hyperexponential functions.

Lemma 11. With Convention 3, we further assume that $r$ is a nonzero residual form w.r.t. $K$. Then the hyperexponential function $r T$ is not integrable.

Proof. Let $\mathcal{M}_{K}$ be the subspace for polynomial reduction, and $\mathcal{N}_{K}$ its standard complement w.r.t. $K$. By the definition of residual forms, there exist $b, q \in \mathbb{E}[y]$ with $b$ being squarefree and $v \in \mathcal{N}_{K}$ s.t.

$$
\operatorname{deg} b>\operatorname{deg} q, \operatorname{gcd}\left(b, k_{2}\right)=1, \text { and } r=\frac{q}{b}+\frac{v}{k_{2}} .
$$

Thus, $r$ can be rewritten as $a /\left(b k_{2}\right)$ for some $a \in \mathbb{E}[y]$. Note that $a$ is not necessarily coprime with $b k_{2}$. It follows that

$$
r T=\frac{a}{b} \exp \left(\int \frac{k_{1}-D_{y}\left(k_{2}\right)}{k_{2}} d y\right) .
$$

Since $\left(k_{1}-D_{y}\left(k_{2}\right)\right) / k_{2}$ is differential-reduced and $k_{2}, b$ are coprime, $\left(a / b,\left(k_{1}-D_{y}\left(k_{2}\right)\right) / k_{2}\right)$ is indecomposable according to Definition 2 in [10]. By Theorem 4 in [10], $a / b$ is in $\mathbb{E}[y]$. So the denominator of $r$ divides $k_{2}$, which, together with (10), implies that $q=0$. Consequently, $\left(v / k_{2}\right) T$ is integrable. By (2), $v=k_{2} D_{y}(s)+k_{1} s$ for some $s \in \mathbb{E}(y)$. Since $v \in \mathbb{E}[y], v \in \mathcal{M}_{K}$ by Lemma 6 (i). Thus, $v=0$ because $v \in \mathcal{N}_{K}$. We have that $r=0$, a contradiction to the assumption that $r \neq 0$.

The existence and uniqueness of residual forms are described below.

Lemma 12. With Convention 3, we have that the shell $S$ has a residual form w.r.t. the kernel $K$. If a rational function has two residual forms w.r.t. $K$, then they are equal.

Proof. By (9), $S=D_{y}\left(S_{1}+w\right)+\left(S_{1}+w\right) K+q / b+v / k_{2}$. So $q / b+v / k_{2}$ is a required form.

Let $r=q / b+v / k_{2}$ and $r^{\prime}=q^{\prime} / b^{\prime}+v^{\prime} / k_{2}$ be two residual forms of a rational function w.r.t. $K$, where $b, b^{\prime}, q, q^{\prime}, v, v^{\prime}$ are in $\mathbb{E}[y], b$ and $b^{\prime}$ are squarefree, $\operatorname{gcd}\left(b, k_{2}\right)=\operatorname{gcd}\left(b^{\prime}, k_{2}\right)=1$, 
$\operatorname{deg} q<\operatorname{deg} b, \operatorname{deg} q^{\prime}<\operatorname{deg} b^{\prime}$ and $v, v^{\prime} \in \mathcal{N}_{K}$. By the definition of residual forms, $D_{y}(f)+f K+r=D_{y}\left(f^{\prime}\right)+f^{\prime} K+r^{\prime}$ for some $f, f^{\prime} \in \mathbb{E}(y)$. It follows that

$$
D_{y}\left(f-f^{\prime}\right)+\left(f-f^{\prime}\right) K+r-r^{\prime}=0 .
$$

Hence, $\left(r^{\prime}-r\right) T$ is integrable by (2). Since $r-r^{\prime}$ is also a residual form w.r.t. $K, r=r^{\prime}$ by Lemma 11 .

Below is the main result of the present section.

Theorem 13. Let $H$ be a hyperexponential function whose logarithmic derivative has kernel $K$ and shell $S$. Then there is an algorithm for computing a rational function $h$ in $\mathbb{E}(y)$ and a unique residual form $r$ w.r.t. $K$ s.t.

$$
H=D_{y}\left(h \exp \left(\int K d y\right)\right)+r \exp \left(\int K d y\right) .
$$

Moreover, $H$ is integrable if and only if $r=0$.

Proof. Let $T=\exp \left(\int K d y\right)$. Applying the shell reduction to $H$ w.r.t. $K$, we can find a rational function $S_{1}$, and two polynomials $a, b$ s.t. (4) holds. Then we apply the polynomial reduction to $a /\left(b k_{2}\right) T$ to get the residual form $r=q / b+v / k_{2}$ s.t. (11) holds.

Suppose that there exists another decomposition

$$
H=D_{y}\left(h^{\prime} T\right)+r^{\prime} T
$$

for some $h^{\prime} \in \mathbb{E}(y)$ and $r^{\prime}$ is a residual form w.r.t. $K$. Then both $r$ and $r^{\prime}$ are residual forms of $S$ by (11), (12) and the fact $H=S T$. So $r=r^{\prime}$ by Lemma 12 .

If $r=0$, then $H$ is obviously integrable. Conversely, assume that $H$ is integrable. Then $r T$ is also integrable by (11). So $r=0$ by Lemma 11 .

The reduction algorithm described in the proof of Theorem 13 has three interesting features. First, it enables us to decide hyperexponential integrability immediately. Second, it decomposes a hyperexponential function into a sum of an integrable one and a non-integrable one in a canonical way. Third, it does not need to compute a polynomial solution of any first-order linear differential equation. The method will be referred to as Hermite reduction for hyperexponential functions in the sequel, because it extends all important conclusions obtained by Hermite reduction for rational functions to hyperexponential ones.

Example 14. Let $H$ be the same hyperexponential function as in Example 2. Then $K=y /\left(y^{2}+1\right)$ and $S=1 /(y-1)^{2}$. Set $T=\sqrt{y^{2}+1}$. By the shell reduction in Example 4 ,

$$
H=D_{y}\left(\frac{-1}{y-1} T\right)+\frac{y}{b k_{2}} T
$$

where $b=y-1$ and $k_{2}=y^{2}+1$. The polynomial reduction yields $\left(y /\left(b k_{2}\right)\right) T=D_{y}(-T / 2)+\left(1 /(2 b)+1 /\left(2 k_{2}\right)\right) T$. Combining the above equations, we decompose $H$ as

$$
H=D_{y}\left(\frac{-(y+1)}{2(y-1)} T\right)+\left(\frac{1}{2 b}+\frac{1}{2 k_{2}}\right) T .
$$

Example 15. Consider $H=y \exp (y)$ as given in Example 5. Since its logarithmic derivative has kernel $K=1$, the subspace $\mathcal{M}_{K}$ of polynomial reduction is equal to $\mathbb{E}[y]$. Thus, $y \in \mathcal{M}_{K}$ and $H$ is integrable. More generally, $\mathcal{M}_{K}=\mathbb{E}[y]$ corresponds to the wellknown fact that $p(y) \exp (y)$ is integrable for all $p \in \mathbb{E}[y] \backslash\{0\}$.

\section{KERNEL REDUCTION}

Let $K=k_{1} / k_{2}$ be a nonzero differential-reduced rational function in $\mathbb{E}(y)$ with $\operatorname{gcd}\left(k_{1}, k_{2}\right)=1$. We may want to reduce a hyperexponential function in the form

$$
\frac{p}{k_{2}^{m}} \exp \left(\int K d y\right) \quad \text { for some } p \in \mathbb{E}[y] \text { and } m \in \mathbb{N} \text {. }
$$

One way would be to rewrite the above function as

$$
p \exp \left(\int \frac{k_{1}-m D_{y}\left(k_{2}\right)}{k_{2}} d y\right),
$$

and proceed by polynomial reduction w.r.t. the new kernel $\left(k_{1}-m D_{y}\left(k_{2}\right)\right) / k_{2}$, which is also differential-reduced. However, it will prove to be more convenient in Section 6 to reduce the given function w.r.t. the initial kernel $K$. To this end, we introduce another type of reduction, based on the ideas in $[9,18]$.

Lemma 16. With Convention 3, we let $p \in \mathbb{E}[y]$ and $m \geq 1$. Then there exist $p_{1}, p_{2} \in \mathbb{E}[y]$ s.t.

$$
\frac{p}{k_{2}^{m}}=D_{y}\left(\frac{p_{1}}{k_{2}^{m-1}}\right)+\frac{p_{1}}{k_{2}^{m-1}} K+\frac{p_{2}}{k_{2}} .
$$

Proof. We proceed by induction on $m$. If $m=1$, then taking $p_{1}=0$ and $p_{2}=p$ yields the claimed form. Assume that $m>1$. We first show that there exist $\tilde{p}_{1}, \tilde{p}_{2} \in \mathbb{E}[y]$ s.t.

$$
\frac{p}{k_{2}^{m}}=D_{y}\left(\frac{\tilde{p}_{1}}{k_{2}^{m-1}}\right)+\frac{\tilde{p}_{1}}{k_{2}^{m-1}} K+\frac{\tilde{p}_{2}}{k_{2}^{m-1}}
$$

which is equivalent to

$$
p=\tilde{p}_{1}\left(k_{1}-(m-1) D_{y}\left(k_{2}\right)\right)+\left(D_{y}\left(\tilde{p}_{1}\right)+\tilde{p}_{2}\right) k_{2} .
$$

Since $k_{1} / k_{2}$ is differential-reduced, there exist $u, v \in \mathbb{E}[y]$ s.t. $p=u\left(k_{1}-(m-1) D_{y}\left(k_{2}\right)\right)+v k_{2}$ by the extended Euclidean algorithm. So we can take $\tilde{p}_{1}=u$ and $\tilde{p}_{2}=v-D_{y}(u)$. By the induction hypothesis, there exist $\bar{p}_{1}, \bar{p}_{2} \in \mathbb{E}[y]$ s.t.

$$
\frac{\tilde{p}_{2}}{k_{2}^{m-1}}=D_{y}\left(\frac{\bar{p}_{1}}{k_{2}^{m-2}}\right)+\frac{\bar{p}_{1}}{k_{2}^{m-2}} K+\frac{\bar{p}_{2}}{k_{2}} \text {. }
$$

Setting $p_{1}=\bar{p}_{1} k_{2}+\tilde{p}_{1}$ and $p_{2}=\bar{p}_{2}$ completes the proof.

With Convention 3, we have

$$
\frac{p}{k_{2}^{m}} T=D_{y}\left(\frac{p_{1}}{k_{2}^{m-1}} T\right)+\frac{p_{2}}{k_{2}} T
$$

by Lemma 16 . This reduction will be referred to as the kernel reduction for $\left(p / k_{2}^{m}\right) T$ w.r.t. $K$.

\section{TELESCOPING VIA REDUCTIONS}

Hermite reduction has been used to construct telescopers for bivariate rational functions in [5]. The goal of this section is to develop a reduction-based telescoping method for bivariate hyperexponential functions.

\subsection{Creative telescoping for bivariate rational functions}

We briefly recall the reduction-based method for rationalfunction telescoping in [5].

Let $\mathbb{F}$ be a field of characteristic zero and $\mathbb{F}(x, y)$ be the field of rational functions in $x$ and $y$ over $\mathbb{F}$. Let $D_{x}$ and $D_{y}$ denote the usual derivations $\partial / \partial x$ and $\partial / \partial y$, respectively. 
Let $\mathbb{F}(x)\left\langle D_{x}\right\rangle$ be the ring of linear differential operators over $\mathbb{F}(x)$. The ring $\mathbb{F}(x)\left\langle D_{x}\right\rangle$ is a left Euclidean domain and its left ideals are principal. For $r \in \mathbb{F}(x, y)$, the telescoping problem is to construct a nonzero linear differential operator $L\left(x, D_{x}\right) \in \mathbb{F}(x)\left\langle D_{x}\right\rangle$ s.t. $L\left(x, D_{x}\right)(r)=D_{y}(s)$, where $s \in \mathbb{F}(x, y)$. The operator $L$ is called a telescoper for $r$, and $s$ is the corresponding certificate. The set $\mathcal{T}$ of all telescopers for a given rational function is a left ideal of $\mathbb{F}(x)\left\langle D_{x}\right\rangle$. Any generator of $\mathcal{T}$ is called a minimal telescoper for the given rational function.

For any $i \in \mathbb{N}$, rational Hermite reduction (w.r.t. $y$ ) decomposes $D_{x}^{i}(r)$ into $D_{x}^{i}(r)=D_{y}\left(s_{i}\right)+a_{i} / b$, where $s_{i} \in \mathbb{F}(x, y)$ and $a_{i}, b \in \mathbb{F}(x)[y]$ with $\operatorname{deg}_{y}\left(a_{i}\right)<\operatorname{deg}_{y}(b)$, and $b$ is squarefree over $\mathbb{F}(x)$. Since $\operatorname{deg}_{y}\left(a_{i}\right)$ is bounded by $\operatorname{deg}_{y}(b)$, the sequence $\left\{a_{i}\right\}_{i \in \mathbb{N}}$ is linearly dependent over $\mathbb{F}(x)$. Assume that there exist $e_{0}, \ldots, e_{\rho} \in \mathbb{F}(x)$, not all zero, s.t. $\sum_{i=0}^{\rho} e_{i} a_{i}=0$. Then $L:=\sum_{i=0}^{\rho} e_{i} D_{x}^{i}$ is a telescoper for $r$ and $\sum_{i=0}^{\rho} e_{i} g_{i}$ is the corresponding certificate. In fact, $L$ is a minimal telescoper for $r$ if $\rho$ is the minimal integer s.t. $e_{0}, \ldots, e_{\rho}$ are linearly dependent over $\mathbb{F}(x)$. This reasoning yields the upper bound $\operatorname{deg}_{y}(b)$ on the order of minimal telescopers.

\subsection{Creative telescoping for bivariate hyper- exponential functions}

We now apply the Hermite reduction for univariate hyperexponential functions in Section 4 to compute telescopers for bivariate hyperexponential functions.

A nonzero element $H$ in some differential field extension of $\mathbb{F}(x, y)$ is said to be hyperexponential over $\mathbb{F}(x, y)$ if its logarithmic derivatives $D_{x}(H) / H$ and $D_{y}(H) / H$ are in $\mathbb{F}(x, y)$.

Put $f=D_{x}(H) / H$ and $g=D_{y}(H) / H$. Then $D_{y}(f)=D_{x}(g)$ because $D_{x}$ and $D_{y}$ commute. Therefore, it is legitimate to denote $H$ by $\exp \left(\int f d x+g d y\right)$. For two hyperexponential functions $H_{i}=\exp \left(\int f_{i} d x+g_{i} d y\right), i=1,2$, we have

$$
H_{1} H_{2}=\exp \left(\int\left(f_{1}+f_{2}\right) d x+\left(g_{1}+g_{2}\right) d y\right) \text {. }
$$

In particular, the product of a rational function $r \in \mathbb{F}(x, y)$ and a hyperexponential function $H=\exp \left(\int f d x+g d y\right)$ is

$$
r H=\exp \left(\int\left(f+D_{x}(r) / r\right) d x+\left(g+D_{y}(r) / r\right) d y\right) .
$$

The following fact is immediate from [10, Lemma 8].

Fact 17. Let $f$ and $g$ be rational functions in $\mathbb{F}(x, y)$ satisfying $D_{y}(f)=D_{x}(g)$. Then the denominator of $f$ divides that of $g$ in $\mathbb{F}(x)[y]$.

For a hyperexponential function $H$ over $\mathbb{F}(x, y)$, the telescoping problem is to construct a linear ordinary differential operator $L\left(x, D_{x}\right)$ in $\mathbb{F}(x)\left\langle D_{x}\right\rangle$ s.t.

$$
L\left(x, D_{x}\right)(H)=D_{y}(G)
$$

for some hyperexponential function $G$ over $\mathbb{F}(x, y)$. As in the rational case, our idea is to apply the Hermite reduction for univariate hyperexponential functions w.r.t. $y$ to the derivatives $D_{x}^{i}(H)$ iteratively, and then find a linear dependency among the residual forms over $\mathbb{F}(x)$.

Lemma 18. Let $H=\exp \left(\int f d x+g d y\right)$ be a hyperexponential function over $\mathbb{F}(x, y)$. Let $K$ be the kernel and $S$ the shell of $g$ w.r.t. $y$. Then, for every $i \in \mathbb{N}$, the $i$-th derivative $D_{x}^{i}(H)$ can be decomposed into

$$
D_{x}^{i}(H)=D_{y}\left(u_{i} T\right)+r_{i} T
$$

where $u_{i} \in \mathbb{F}(x, y), T=\exp \left(\int\left(f-D_{x}(S) / S\right) d x+K d y\right)$ and $r_{i} \in \mathbb{F}(x, y)$ is a residual form w.r.t. $K$. Moreover, let $k_{2}$ be the denominator of $K, b$ the squarefree part of the denominator of $S$, and $\mathcal{N}_{K}$ the standard complement of the subspace for polynomial reduction w.r.t. $K$. Then

$$
r_{i}=\frac{q_{i}}{b}+\frac{v_{i}}{k_{2}}
$$

for some $q_{i} \in \mathbb{F}(x)[y]$ with $\operatorname{deg}_{y} q_{i}<\operatorname{deg}_{y} b$ and $v_{i} \in \mathcal{N}_{K}$.

Proof. We proceed by induction on $i$. If $i=0$, then the assertion holds by Theorem 13 .

Assume that $D_{x}^{i}(H)$ can be decomposed into (14) and assume that (15) holds. Moreover, let $\tilde{f}=f-D_{x}(S) / S$. Consider the $(i+1)$-th derivative $D_{x}^{i+1}(h)$. There exists a polynomial $a$ in $\mathbb{F}(x)[y]$ s.t. $\tilde{f}=a / k_{2}$ by $D_{y}(\tilde{f})=D_{x}(K)$ and Fact 17. A direct calculation leads to

$$
\begin{aligned}
D_{x}^{i+1}(H)= & D_{y}\left(D_{x}\left(u_{i} T\right)\right)+\left(\frac{a q_{i}}{b k_{2}}+\frac{D_{x}\left(q_{i}\right)}{b}+\frac{D_{x}\left(v_{i}\right)}{k_{2}}\right) T \\
& +\left(\frac{-q_{i} D_{x}(b)}{b^{2}}+\frac{\left(a-D_{x}\left(k_{2}\right)\right) v_{i}}{k_{2}^{2}}\right) T .
\end{aligned}
$$

Applying the shell reduction to $\left(-q_{i} D_{x}(b) / b^{2}\right) T$ and the kernel reduction to $\left(\left(a-D_{x}\left(k_{2}\right)\right) v_{i} / k_{2}^{2}\right) T$ w.r.t. $y$, we get

$$
\begin{aligned}
\frac{-q_{i} D_{x}(b)}{b^{2}} & =D_{y}\left(\frac{w_{1}}{b}\right)+\frac{w_{1}}{b} K+\frac{w_{2}}{b k_{2}}, \\
\frac{\left(a-D_{x}\left(k_{2}\right)\right) v_{i}}{k_{2}^{2}} & =D_{y}\left(\frac{p_{1}}{k_{2}}\right)+\frac{p_{1}}{k_{2}} K+\frac{p_{2}}{k_{2}},
\end{aligned}
$$

where $w_{1}, w_{2}, p_{1}$ and $p_{2}$ are in $\mathbb{F}(x)[y]$. We then apply polynomial reduction to $\tilde{S} T$ w.r.t. $K$, where

$$
\tilde{S}=\frac{w_{2}}{b k_{2}}+\frac{p_{2}}{k_{2}}+\frac{a q_{i}}{b k_{2}}+\frac{D_{x}\left(q_{i}\right)}{b}+\frac{D_{x}\left(v_{i}\right)}{k_{2}},
$$

which leads to

$$
\tilde{S}=D_{y}(w)+w K+\left(\frac{q_{i+1}}{b}+\frac{v_{i+1}}{k_{2}}\right),
$$

where $w \in \mathbb{F}(x, y)$ and $q_{i+1} / b+v_{i+1} / k_{2}$ is the residual form of $\tilde{S}$ w.r.t. $K$. It follows from a direct calculation that

$$
D_{x}^{i+1}(H)=D_{y}\left(u_{i+1} T\right)+\left(\frac{q_{i+1}}{b}+\frac{v_{i+1}}{k_{2}}\right) T,
$$

where $u_{i+1}=D_{x}\left(u_{i}\right)+u_{i} \tilde{f}+w_{1} / b+p_{1} / k_{2}+w$.

The main results in the present section are given below.

Theorem 19. With the notation introduced in Lemma 18, we let $L=\sum_{i=0}^{\rho} e_{i} D_{y}^{i}$ with $e_{0}, \ldots, e_{\rho} \in \mathbb{F}(x)$, not all zero.

(i) $L$ is a telescoper for $H$ if and only if $\sum_{i=1}^{\rho} e_{i} r_{i}=0$.

(ii) The order of a minimal telescoper for $H$ is no more than $\operatorname{deg}_{y}(b)+\max \left(\operatorname{deg}_{y}\left(k_{1}\right), \operatorname{deg}_{y}\left(k_{2}\right)-1\right)$.

Proof. We set $\mathbb{E}=\mathbb{F}(x)$ and view that hyperexponential functions involved in the proof are over $\mathbb{E}(y)$. Moreover, let $u=\sum_{i=0}^{\rho} e_{i} u_{i}$ and $r=\sum_{i=0}^{\rho} e_{i} r_{i}$. By (14), we have

$$
L(H)=D_{y}(u T)+r T .
$$

If $r=0$, then $L$ is a telescoper by (16). Conversely, assume that $L$ is a telescoper of $h$. Then $r T$ is integrable w.r.t. $y$ 
by (16). Since $r$ is a residual form, it is equal to zero by Lemma 11. The first assertion is proved.

Set $\lambda=\max \left(\operatorname{deg}_{y}\left(k_{1}\right), \operatorname{deg}_{y}\left(k_{2}\right)-1\right)$. Let the residual form $r_{i}=q_{i} / b+v_{i} / k_{2}$ be as defined in (14) and (15). By Corollary 9 , the $v_{i}$ 's have a common set $\mathcal{P}$ of supporting monomials with $|\mathcal{P}| \leq \lambda$. Moreover, $\operatorname{deg}_{y}\left(q_{i}\right)<\operatorname{deg}_{y}(b)$ and $\operatorname{gcd}\left(b, k_{2}\right)=1$. Therefore, the residual forms $r_{0}, \ldots, r_{\rho}$ are linearly dependent over $\mathbb{F}(x)$ if $\rho \geq \operatorname{deg}_{y}(b)+\lambda$. The second assertion holds

Remark 20. By Theorem 19, the first linear dependency among the residual forms $r_{0}, r_{1}, r_{2}, \ldots$ gives rise to a minimal telescoper of $H$.

Below is an outline of the reduction based telescoping algorithm for hyperexponential functions, in which the notation is that introduced in Lemma 18 is used.

Algorithm. HermiteTelescoping: Given a bivariate hyperexponential function $H=\exp \left(\int f d x+g d y\right)$ over $\mathbb{F}(x, y)$, compute a minimal telescoper $L$ and its certificate w.r.t. $y$.

1. Find the kernel $K$ and shell $S$ of $D_{y}(H) / H$ w.r.t. $y$. Set $b$ to be the squarefree part of the denominator of $S$.

2. Decompose $H$ into $H=D_{y}\left(u_{0} T\right)+r_{0} T$ using the Hermite reduction for hyperexponential functions given in Theorem 13. If $r_{0}=0$, return $\left(1, u_{0} T\right)$.

3. Set $\rho:=\operatorname{deg}_{y}(b)+\max \left(\operatorname{deg}_{y}\left(k_{1}\right), \operatorname{deg}_{y}\left(k_{2}\right)-1\right)$.

4. For $i$ from 0 to $\rho$ do

4.1. Compute $\left(u_{i}, r_{i}\right)$ incrementally s.t.

$$
D_{x}^{i}(H)=D_{y}\left(u_{i} T\right)+r_{i} T
$$

by the shell, kernel and polynomial reductions described in Lemma 18.

4.2. Find $\eta_{j} \in \mathbb{F}(x)$ s.t. $\sum_{j=0}^{i} \eta_{j} r_{j}=0$ using the algorithm in [17]. If there is a nontrivial solution, $\operatorname{return}\left(\sum_{j=0}^{i} \eta_{j} D_{x}^{j}, \sum_{j=0}^{i} \eta_{j} u_{j} T\right)$.

Example 21. Let $H=\sqrt{x-2 y} \exp \left(x^{2} y\right)$. Then $D_{x}(H) / H$ and $D_{y}(H) / H$ are, respectively,

$$
f=\frac{1+4 x^{2} y-8 x y^{2}}{2(x-2 y)} \quad \text { and } g=\frac{-1+x^{3}-2 x^{2} y}{x-2 y} .
$$

Since $g$ is differential-reduced w.r.t. $y, g$ is the kernel and 1 is the shell of $D_{y}(H) / H$ w.r.t. y. By Hermite reduction,

$$
H=D_{y}\left(\frac{1}{x^{2}} H\right)+\frac{1}{x^{2} k_{2}} H .
$$

Applying $D_{x}$ to the above equation yields

$$
D_{x}(H)=D_{y}\left(\frac{-3 x+8 y+4 x^{3} y-8 x^{2} y^{2}}{2 x^{3}(x-2 y)} H\right)+r H,
$$

where $r=\left(-5 x+8 y+4 x^{3} y-8 x^{2} y^{2}\right) /\left(2 x^{3} k_{2}^{2}\right)$. The shell, kernel and polynomial reduction given in Lemma 18 yields

$$
D_{x}(H)=D_{y}\left(\frac{2 x^{2} y-3}{x^{3}} \cdot H\right)+\frac{3 x^{3}-6}{2 x^{3} k_{2}} H
$$

Combining (17) and (18), we get $L=\left(6-3 x^{3}\right)+2 x D_{x}$ is a minimal telescoper for $H$ and $G=(4 y-3 x) H$ is the corresponding certificate.
Remark 22. The algorithm HermiteTelescoping is directly based on the proof of Lemma 18. Yet, there is another idea for computing a minimal telescoper of $H$. Namely, we first compute a nonzero operator $L_{1} \in \mathbb{F}(x)\left\langle D_{x}\right\rangle$ of minimal order s.t. $L_{1}(H)=D_{y}\left(G_{1}\right)+\left(p / k_{2}\right) T$ for some hyperexponential function $G_{1}$ and polynomial p. Note that such operators always exist, because $\operatorname{deg}_{y} q_{i}$ in (15) is less than $\operatorname{deg}_{y} b$. Then we apply the algorithm HermiteTelescoping to get a minimal telescoper $L_{2}$ for $\left(p / k_{2}\right) T$. In doing so, any rational function with denominator $b$ will not appear when we compute $L_{2}$. It turns out that $L_{2} L_{1}$ is a minimal telescoper of $H$. An implementation on this idea is underway.

\subsection{Comparison with the Apagodu-Zeilberger bound}

Assume that

$$
H=u \exp \left(\frac{r_{1}}{r_{2}}\right) \prod_{i=1}^{m} p_{i}(x, y)^{c_{i}}
$$

where $u, r_{1}, r_{2}, p_{1}, \ldots, p_{m}$ are nonzero polynomials in $\mathbb{F}[x, y]$ and $c_{1}, \ldots, c_{m}$ are distinct indeterminates. Theorem cAZ in [4] asserts that the order of minimal telescopers for $H$ is bounded by

$\alpha:=\operatorname{deg}_{y}\left(r_{2}\right)+\max \left(\operatorname{deg}_{y}\left(r_{1}\right), \operatorname{deg}_{y}\left(r_{2}\right)\right)+\sum_{i=1}^{m} \operatorname{deg}_{y}\left(p_{i}\right)-1$.

Note that $H$ can be viewed as a hyperexponential function over $\mathbb{F}\left(c_{1}, \ldots, c_{m}\right)(x, y)$. We now show that $\alpha$ given above is no less than the order bound on minimal telescopers for $H$ obtained from Theorem 19 (ii). The kernel and shell of the logarithmic derivative $D_{y}(H) / H$ are

$$
K:=D_{y}\left(\frac{r_{1}}{r_{2}}\right)+\sum_{i=1}^{m} c_{i} \frac{D_{y}\left(p_{i}\right)}{p_{i}} \quad \text { and } \quad S:=u,
$$

respectively, because $K$ has no integral residue at any simple pole, $S$ is a polynomial in $\mathbb{F}[x, y]$, and $D_{y}(H) / H$ is equal to $K+D_{y}(S) / S$. Let $K=k_{1} / k_{2}$ with $\operatorname{gcd}\left(k_{1}, k_{2}\right)=1$. A direct calculation leads to

$$
\operatorname{deg}_{y}\left(k_{1}\right) \leq \operatorname{deg}_{y}\left(r_{1}\right)+\operatorname{deg}_{y}\left(r_{2}\right)+\sum_{i=1}^{m} \operatorname{deg}_{y}\left(p_{i}\right)-1,
$$

and

$$
\operatorname{deg}_{y}\left(k_{2}\right) \leq 2 \operatorname{deg}_{y}\left(r_{2}\right)+\sum_{i=1}^{m} \operatorname{deg}_{y}\left(p_{i}\right)
$$

By Theorem 19, the order of minimal telescopers for $H$ is no more than $\max \left(\operatorname{deg}_{y}\left(k_{1}\right), \operatorname{deg}_{y}\left(k_{2}\right)-1\right)$, which is no more than $\alpha$ by the above two inequalities.

Indeed, the order bound in Theorem 19 (ii) may be smaller than that in Theorem cAZ.

Example 23. Let $H=q^{c} \exp (a / q)$, where $a, q$ are irreducible polynomials in $\mathbb{F}[x, y]$ with $\operatorname{deg}_{y}(a)<\operatorname{deg}_{y}(q)$, and $c$ is a transcendental constant over $\mathbb{F}$. By Theorem $c A Z$, a minimal telescoper for $H$ has order no more than $3 \operatorname{deg}_{y} q-1$. On the other hand, the kernel and shell of $D_{y}(H) / H$ are equal to $\left(D_{y}(a) q-a D_{y}(q)+c q D_{y}(q)\right) / q^{2}$ and 1 , respectively. A minimal telescoper has order no more than $2 \operatorname{deg}_{y} q-1$ by Theorem 19 (ii). 
In general, Christopher's Theorem states that a hyperexponential function over $\mathbb{F}(x, y)$ can always be written as:

$$
\frac{u}{v} \exp \left(\frac{r_{1}}{r_{2}}\right) \prod_{i=1}^{m} p_{i}(x, y)^{c_{i}},
$$

where $u, v, r_{1}, r_{2} \in \mathbb{F}[x, y], c_{i}$ is algebraic over $\mathbb{F}$, and $p_{i}$ is in $\mathbb{F}\left(c_{i}\right)[x, y], i=1, \ldots, m$. A more explicit description on (20) can be found in [7]. So $H$ given in (19) is a special instance for hyperexponential functions. In addition, it is easier to compute the kernel and shell than to compute the decompositions (19) and (20) when a hyperexponential function is given by its logarithmic derivatives.

\section{IMPLEMENTATION AND TIMINGS}

We have produced a preliminary implementation of the algorithm HermiteTelescoping in the computer algebra system Maple 16. Our Maple code is available from

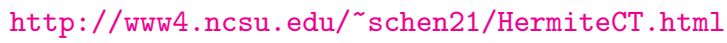

We now compare the performance of our algorithm to the Maple function DEtools[Zeilberger] of the telescoping algorithm in [3]. The examples for comparison are of the form

$$
\frac{p}{q^{m}} \cdot \sqrt{\frac{a}{b}} \cdot \exp \left(\frac{u}{v}\right)
$$

where $m \in \mathbb{N}, p, q, a, b, u, v \in \mathbb{Z}[x, y]$ are irreducible and their coefficients are randomly chosen. For simplicity, we choose $\lambda=\operatorname{deg}_{y}(p)=\operatorname{deg}_{y}(q), \mu=\operatorname{deg}_{y}(a)=\operatorname{deg}_{y}(b)$, and $\nu=\operatorname{deg}_{y}(u)=\operatorname{deg}_{y}(v)$. The runtime comparison (in seconds) for different examples is shown in Table 1 , in which

- ZT: the Maple function DEtools[Zeilberger].

- HT: our implementation of HermiteTelescoping.

- order: the order of the computed minimal telescoper.

- OOM: Maple runs out of memory.

\begin{tabular}{c|r|r|c}
$(\lambda, \mu, \nu, m)$ & ZT & HT & order \\
\hline$(2,0,2,1)$ & 2.23 & 2.43 & 5 \\
$(2,0,2,2)$ & 2.21 & 2.01 & 5 \\
$(3,0,2,1)$ & 8.72 & 6.64 & 6 \\
$(3,0,2,2)$ & 9.38 & 6.56 & 6 \\
$(6,0,1,1)$ & 45.35 & 24.49 & 7 \\
$(6,0,1,2)$ & 43.02 & 22.91 & 7 \\
$(2,2,2,1)$ & 1405.9 & 221.5 & 9 \\
$(2,2,2,2)$ & 1398.1 & 200.34 & 9 \\
$(3,0,3,1)$ & 147.92 & 47.23 & 8 \\
$(3,0,3,2)$ & 151.20 & 44.56 & 8 \\
$(3,3,0,1)$ & 207.82 & 61.10 & 8 \\
$(3,3,0,2)$ & 211.70 & 58.63 & 8 \\
$(3,2,1,1)$ & 304.61 & 62.67 & 8 \\
$(3,2,1,2)$ & 331.0 & 63.61 & 8 \\
$(3,1,3,1)$ & OOM & 534.87 & 10 \\
$(3,1,3,2)$ & OOM & 522.15 & 10 \\
\hline
\end{tabular}

Table 1: Timings (in sec.) were taken on a Mac OS $\mathrm{X}$ computer with $4 \mathrm{~Gb} \mathrm{RAM}$ and $3.06 \mathrm{GHz}$ Core 2 Duo processor.

Remark 24. The orders of the computed minimal telescopers in our experiments are equal to the predicted order bounds in Theorem 19.

\section{REFERENCES}

[1] S.A. Abramov. The rational component of the solution of a first order linear recurrence relation with rational right hand-side. Ž. Vyčisl. Mat. i Mat. Fiz., 15(4):1035-1039, 1090, 1975.

[2] S.A. Abramov and M. Petkovšek. Minimal decomposition of indefinite hypergeometric sums. In ISSAC'01: Proceedings of the 2001 International Symposium on Symbolic and Algebraic Computation, pages 7-14, New York, 2001. ACM.

[3] G. Almkvist and D. Zeilberger. The method of differentiating under the integral sign. J. Symbolic Comput., 10:571-591, 1990.

[4] M. Apagodu and D. Zeilberger. Multi-variable Zeilberger and Almkvist-Zeilberger algorithms and the sharpening of Wilf- Zeilberger theory. Adv. in Appl. Math., 37(2):139-152, 2006.

[5] A. Bostan, S. Chen, F. Chyzak, and Z. Li. Complexity of creative telescoping for bivariate rational functions. In ISSAC'10: Proceedings of the 2010 International Symposium on Symbolic and Algebraic Computation, pages 203-210, New York, NY, USA, 2010. ACM.

[6] M. Bronstein. Symbolic Integration I: Transcendental Functions, volume 1 of Algorithms and Computation in Mathematics. Springer-Verlag, Berlin, second edition, 2005.

[7] S. Chen. Some applications of differential-difference algebra to creative telescoping. PhD thesis, École Polytechnique (Palaiseau, France), February 2011.

[8] C. Christopher. Liouvillian first integrals of second order polynomial differential equations. Electron. J. Differential Equations, 49:1-7, 1999.

[9] J.H. Davenport. The Risch differential equation problem. SIAM J. Comput., 15(4):903-918, 1986.

[10] K.O. Geddes, H.Q. Le, and Z. Li. Differential rational normal forms and a reduction algorithm for hyperexponential functions. In ISSAC'04: Proceedings of the 2004 International Symposium on Symbolic and Algebraic Computation, pages 183-190, New York, USA, 2004. ACM.

[11] R.W. Gosper, Jr. Decision procedure for indefinite hypergeometric summation. Proc. Nat. Acad. Sci. U.S.A., 75(1):40-42, 1978.

[12] C. Hermite. Sur l'intégration des fractions rationnelles. Ann. Sci. École Norm. Sup. (2), 1:215-218, 1872.

[13] E. Horowitz. Algorithms for partial fraction decomposition and rational function integration. In $S Y M S A C^{\prime}$ '1, pages 441-457, New York, USA, 1971. ACM.

[14] M. Kauers, C. Koutschan, and D. Zeilberger. Proof of Ira Gessel's lattice path conjecture. Proc. Natl. Acad. Sci. USA, 106(28):11502-11505, 2009.

[15] C. Koutschan, M. Kauers, and D. Zeilberger. Proof of George Andrews's and David Robbins's $q$-TSPP conjecture. Proc. Natl. Acad. Sci. USA, 108(6):2196-2199, 2011.

[16] M.V. Ostrogradski1̌. De l'intégration des fractions rationnelles. Bull. de la classe physico-mathématique de l'Acad. Impériale des Sciences de Saint-Pétersbourg, 4:145-167, 286-300, 1845.

[17] A. Storjohann and G. Villard. Computing the rank and a small nullspace basis of a polynomial matrix. In ISSAC'05: Proceedings of the 2005 International Symposium on Symbolic and Algebraic Computation, pages 309-316. ACM, New York, 2005.

[18] G. Xin and T.Y.J. Zhang. Enumeration of bilaterally symmetric 3-noncrossing partitions. Discrete Math., 309(8):2497-2509, 2009. 